\title{
Boric Acid Vaginal Suppositories: A Brief Review
}

\author{
S.M. Prutting and J.D. Cerveny* \\ Ambulatory Care, Medical College of South Carolina, Charleston, SC
}

KEY WORDS

boric acid; recurrent vulvovaginal candidiasis; vaginitis

\section{STRUCTURE AND DERIVATION}

$\mathrm{B}_{\mathrm{a}}^{\mathrm{o}}$ oric acid, also called boracic acid or orthoboric acid, is an inorganic acid with the chemical formula $\mathrm{H}_{3} \mathrm{BO}_{3}$. It is available as a white, odorless powder and in crystalline and granular forms. ${ }^{1}$ Boric acid vaginal suppositories are not commercially available and, therefore, must be compounded. Most studies documenting efficacy utilize $600 \mathrm{mg}$ of boric acid powder in a gelatin capsule, although other extemporaneous formulations have been developed. . $^{2,3}$

\section{MECHANISM OF ACTION}

Boric acid is a weak, topical, bacteriostatic, and fungistatic agent; however, the exact mechanism of action is unclear. ${ }^{4}$ It has been suggested that the fungistatic activity may be mediated by vaginal acidification, resulting in fungal cell wall penetration and disruption of the fungal cell membrane. ${ }^{5}$ Conversely, studies evaluating the minimum inhibitory concentration of boric acid indicate that boric acid works at a $\mathrm{pH}$ similar to that of the untreated vaginal tract, and, therefore, the action may not be simply due to an increase in acidity. ${ }^{6,7}$

\section{PHARMACOKINETICS}

Boric acid is rapidly and completely absorbed following oral ingestion and is well absorbed through denuded and abraded skin in solution or as a dry powder. ${ }^{8}$ Absorption has been reported to be negligible through intact skin in the presence of alkaline salts. ${ }^{9}$ Once absorbed, boric acid is widely distributed throughout body water and accumulates in the brain, liver, and kidneys. ${ }^{8}$ Boric acid is not ap- preciably metabolized and is primarily excreted unchanged by the kidneys, with about $50 \%$ excreted within twelve hours of administration ${ }^{10}$ and $90 \%$ of excretion occurring within 96 hours. ${ }^{11}$ Minimal amounts are excreted in feces, sweat, and saliva. ${ }^{11}$ In a study evaluating vaginal absorption of boric acid following the intravaginal administration of one to two 600-mg boric acid capsules for one to two weeks in eight healthy volunteers, daily blood boron concentrations of less than $1 \mu \mathrm{g} / \mathrm{mL}$ during use (mean level, $42 \mu \mathrm{g} / \mathrm{mL}$ ) were reported. ${ }^{12}$ No volunteer had detectable boric acid levels 48 hours after the end of treatment. Boron blood levels below $200 \mu \mathrm{g} / \mathrm{mL}$ are thought to be safe by many investigators, with normal blood boron levels ranging from 0.1 to $80 \mu \mathrm{g} / \mathrm{mL} .{ }^{12,13}$ Blood boron analysis following vaginal insertion of a single $600-\mathrm{mg}$ boric acid capsule in one healthy volunteer revealed systemic boric acid absorption of approximately $6 \%$ from the vagina and a serum half-life of about 10.5 hours. ${ }^{7} \mathrm{~A}$ half life of 21 hours has been reported following administration of a $600-\mathrm{mg}$ intravenous bolus to eight healthy males. ${ }^{11}$

\section{SIDE EFFECTS AND INTERACTIONS}

Short courses of intravaginal boric acid are generally well tolerated. The adverse effects reported most frequently are mild and include watery discharge, erythema, and a burning sensation; also, a partner reported a gritty sensation with intercourse during the treatment period. 5,7,14,15 Although intravaginal boric acid has been reported to be safe when used for short periods of time, long-term safety data is lacking. Additionally, its safety profile

*Correspondence to: Joli D. Cerveny, Clinical Coordinator, Ambulatory Care, Medical College of South Carolina, 590 MUSC, Suite 820R, Charleston, SC 29425. E-mail: cervenyj@musc.edu 
is controversial due to toxicities following ingestion or topical exposure. In the year 1888, three cases of acute poisoning were reported following intravaginal application. ${ }^{16}$ Other reports of vaginally administered boric acid in more than 2,000 patients reveal an absence of toxicity. ${ }^{7}$

The risk of systemic toxicity depends on the concentration used, route of administration, age of the patient, skin condition, and duration of exposure. The fatal adult dose is thought to be about 20 grams or $0.1-0.5 \mathrm{mg} / \mathrm{kg}$ taken orally. ${ }^{8}$ Boric acid poisoning affects multiple organ systems including the gastrointestinal tract, central nervous system (GNS), skin, liver, and kidneys. Vomiting, diarrhea, and abdominal pain are the most common symptoms. Excitement of the CNS followed by malaise, lethargy, headache, seizures, coma, and hyperpyrexia also occurs. Additionally, an erythematous, vesicular, or papular rash with desquamatization, referred to as boiled lobster syndrome, may be observed. The kidneys are probably the most seriously affected of all the organs involved, resulting in renal tubular necrosis, anuria, and albuminuria. Death resulting from cardiovascular collapse, shock, or respiratory failure may occur within 3-5 days of acute poisoning. ${ }^{8,9}$ The risk of systemic toxicity due to vaginal administration of boric acid is minimal. ${ }^{7}$

Symptoms of chronic intoxication include anorexia, gastrointestinal disturbances, weakness, confusion, dermatitis, menstrual disorders, anemia, seizures, and alopecia. No drug-drug interactions have been reported involving boric acid. ${ }^{8,9}$

\section{SPECTRUM OF ACTIVITY}

Early reports of in vitro testing indicate that boric acid has some activity against staphylococci and streptococci, although the suggested action is primarily bacteriostatic and slow-acting. ${ }^{9}$ The use of boric acid in the preservation of urine samples has also been described. ${ }^{17,18}$ Boric acid is most often associated with antifungal activity, although further elucidation is necessary. In vitro studies of boric acid concentrations ranging from $0.4-5.0 \%$ have been demonstrated to inhibit clinical isolates of Candida albicans and $500 \mathrm{mg}$ of boric acid has reportedly killed 50-90\% of $C$. albicans isolates in 48 hours. ${ }^{7,14,19} \mathrm{C}$. albicans is the most common cause of vaginal candidiasis, although non- $C$. albicans species are becoming more frequently encountered as causative agents. ${ }^{20}$ Unfortunately, azole antifungal agents (e.g., fluconazole, itraconazole) appear to be less effective in treating non- $C$. albicans species. ${ }^{21}$ Although no direct comparative trials exist, boric acid may have improved activity against non- $C$. albicans species.

Candida glabrata, formerly called Torulopsis glabrata, is the most common of the non- $C$. albicans species. ${ }^{15,22}$ Many isolates of $G$. glabrata are resistant to azole agents. Boric acid has been demonstrated to be active against $C$. glabrata isolates that are resistant to azoles, although the data is limited. ${ }^{15,22,23}$

Boric acid may also possess antifungal activity against Saccharomyces cerevisiae. ${ }^{24}$ Vaginitis due to this organism is extremely rare, ${ }^{24}$ although colonization of the respiratory and urinary tracts may occur with underlying disease.

Other non-G. albicans species against which boric acid may possess activity include Candida parapsilosis, Candida krusei, Candida tropicalis, and Trichosporon beigelii; however, data supporting its use is limited, and no evidence of susceptibility patterns exist. 5,21,24 Additionally, one report suggests that boric acid is effective in treating azole-resistant $C$. albicans. ${ }^{25}$ Borate salts have also been suggested to inhibit replication and cytopathic effects of the herpes virus; however, the mechanism is not well understood. ${ }^{26}$

\section{CLINICAL APPLICATIONS}

Boric acid vaginal suppositories should not be considered for the first-line treatment of uncomplicated vulvovaginal candidiasis because of insufficient efficacy data and controversies surrounding its safety. Safety and efficacy of the azoles are welldocumented. Boric acid may be considered as an alternative in azole-resistant monilial strains or refractory cases of chronic, recurrent vulvovaginal candidiasis.

Boric acid should not be used in pregnant women due the risk of teratogenic effects and the lack of data demonstrating safety. ${ }^{27}$ No data is available regarding the safety of boric acid in lactating mothers; therefore, it should not be recommended in this patient population.

\section{Resistant Candidal Vaginitis}

Several cases using boric acid for the treatment of azole-refractory candidal vaginitis have been re- 
ported. Shinohara and Tasker ${ }^{5}$ described a case in which a 32-year old female with advanced acquired immunodeficiency syndrome (AIDS) and a CD4 count of $10 / \mathrm{mm} 3$, developed vulvovaginal candidiasis and thrush after nine months of daily treatment with $100 \mathrm{mg}$ of fluconazole. Cultures were positive for $C$. krusei and $C$. glabrata that were resistant to fluconazole. The patients symptoms were not controlled by itraconazole, nystatin only minimally improved her symptoms, and gentian violet topical solution was cosmetically unacceptable to the patient. Treatment with boric acid vaginal suppositories, $600 \mathrm{mg}$ twice daily, and 5\% boric acid in lanolin resulted in relief of symptoms and was well tolerated. Boric acid was not detectable in the serum. Five months following the initial treatment, the patient experienced three relapses that were managed with two- to three-day courses of boric acid.

A second case of fluconazole-resistant $C$. albicans was described by Sobel and Vazquez. ${ }^{25}$ A 38-year old immunocompetent female with a three-month history of $C$. albicans vulvovaginitis previously treated with various over-the-counter topicals, terconazole, and fluconazole developed $C$. albicans that was resistant to fluconazole, itraconazole, and ketoconazole. The patient received treatment with 600 -mg boric acid vaginal capsules taken twice daily for two weeks, resulting in clinical and mycological cure. Redondo-Lopez et al. ${ }^{23}$ described a case of flucytosine-resistant $C$. glabrata in a 30 -year old female who had received several courses of clotrimazole, ketoconazole, miconazole, flucytosine, and intravenous amphotericin B without clinical or mycological response. An immediate clinical and mycologic cure was observed with the initiation of vaginal boric acid capsules, $600 \mathrm{mg}$ twice a day. Following a maintenance course of boric acid for three months, the patient remained without symptoms or positive culture for one year.

\section{Vaginitis due to Saccharomyces cerevisiae}

Boric acid vaginal suppositories may be useful in the treatment of vulvovaginitis caused by Saccharomyces cerevisiae, although these infections are quite rare. Sobel et al. ${ }^{24}$ described a case of a 30 year old female with a five-month history of recurrent vaginitis from which $G$. glabrata was isolated. The patient failed to respond to treatment with clotrimazole, terconazole, ketoconazole, intrave- nous amphotericin B, or oral flucytosine. Clinical and mycological cure was eventually attained with vaginal boric acid suppositories at a dose of $600 \mathrm{mg}$ daily. While still receiving boric acid, the patient was found to be colonized with $S$. cerevisiae and developed frank vulvovaginitis that improved when the dose of boric acid was increased to 600 mg twice daily. The patient remained asymptomatic with boric acid maintenance therapy for six months. Relapse following cessation of therapy was eradicated with boric acid suppositories. Additionally, the authors evaluated the susceptibility of 20 clinical isolates of $S$. cerevisiae, and prolonged therapy with boric acid, $600 \mathrm{mg} / \mathrm{day}$, was associated with clinical resolution and mycologic resolution in four episodes, although mycologic clearance required prolonged therapy.

\section{Vaginitis due to Non-C. albicans Species}

A retrospective review of 75 symptomatic episodes attributed to $C$. glabrata conducted by Sobel and Chaim $^{15}$ reported that $C$. glabrata vaginal infection was associated with a variable but low rate of response to azole therapy, and the use of boric acid resulted in an improved therapeutic response in patients with symptomatic $C$. glabrata vaginitis. The investigators indicated that $600 \mathrm{mg}$ boric acid administered once daily for 14 days resulted in mycological eradication in $77 \%$ of episodes and in clinical cure or improvement in $81 \%$ of episodes.

In a prospective observational study evaluating the importance of fungal cultures in patients with symptoms of chronic vaginitis, Nyirjesy et al. ${ }^{21}$ reported that $32 \%$ of isolates obtained from 77 isolates were non- $C$. albicans. Fluconazole provided short-term mycologic cure in only $25 \%$ of the reported non-C. albicans cases, and boric acid vaginal suppositories, $600 \mathrm{mg}$ twice daily for two weeks, achieved the highest mycologic cure rate. Spinillo et al. ${ }^{28}$ reported that use of boric acid to treat 12 patients with recurrent vulvovaginitis caused by $C$. glabrata yielded good long-term results, with eight patients free of recurrence at 12-month follow-up.

Boric acid has not been extensively studied as a treatment for fungal vaginitis. Several investigators who used boric acid therapy for $C$. albicans reported cure rates of greater than $90 \%, 7,15,29$ although this is not considered first-line therapy due to the availability of shorter, safer treatment modalities. 


\section{COST}

Self-made boric acid capsules (boric acid, USP, and number 0 gelatin capsules) are very inexpensive, with an average wholesale price for a two-week course of treatment of approximately $\$ 0.30 .^{30}$ The actual cost to the patient for compounded capsules or extemporaneously prepared suppositories may range from $\$ 10$ to $\$ 25$ depending on the pharmacy.

\section{CONCLUSIONS}

Boric acid should not be considered first-line treatment for vulvovaginitis due to $C$. albicans as more safe, effective, and shorter duration treatment modalities are available. It may useful, however, in the treatment of chronic recurrent vulvovaginal candidiasis due to azole-resistant strains or treatment failures.

\section{REFERENCES}

1. Budavari S: The Merck Index: An Encyclopedia of Chemicals, Drugs, and Biologicals. 12th ed. Whitehouse Station, NJ: Merck \& Co., Inc., 1996: 219.

2. Allen LV: Boric acid suppositories. US Pharm 21:92-93, 1996.

3. Pesko LJ: Compounding: boric acid antifungal. Am Drug 206:73, 1992.

4. Gosselin RE, Smith RP, Hodge HC: Clinical Toxicology of Commercial Products. 5th ed. Baltimore, MD: Williams \& Wilkins, 1984: III-66-III-69.

5. Shinohara YT, Tasker SA: Successful use of boric acid to control azole-refractory Candida vaginitis in a woman with AIDS. J Acquir Immun Defic Syndromes Hum Retrovirology 16:219-220, 1997.

6. Anonymous: A household remedy for candidiasis. Emergency Med 14: 97-99, 1982.

7. Van Slyke KK, Michel VP, Rein MF: Treatment of vulvovaginal candidiasis with boric acid powder. Am J Obstet Gynecol 141:145-148, 1981.

8. Seigel E, Wason S: Boric acid toxicity. Ped Clin North Am 33:363-367, 1986.

9. Scillinger BM, Berstein M, Goldberg LA, Shalita AR: Boric acid poisoning. J Am Acad Dermatol 7:667-673, 1982.

10. Reynolds JEF: Martindale: The Extra Pharmacopoeia. 31st ed. London: Royal Pharmaceutical Society, 1996: 1680-1681.

11. Jansen JA, Anderson J, Schou JS: Boric acid single dose pharmacokinetics after intravenous administration to man. Arch Toxicol 55:64-67, 1984.
12. Van Slyke KK, Michel VP, Rein MF: The boric acid powder treatment of vulvovaginal candidiasis. College Health 30:107-109, 1981.

13. Pascher F: Systemic reactions to topically applied drugs. Bull NY Acad Med 49:622-623, 1973.

14. Swate TE, Weed JC: Boric acid treatment of vulvovaginal candidiasis. Obstet Gynecol 43:893-895, 1974.

15. Sobel JD, Chaim W: Treatment of Torulopsis glabrata vaginitis: retrospective review of boric acid therapy. Clin Infect Dis 24:649-652, 1997.

16. Valdes-Dapena MA, Arey JB: Boric acid poisoning. J Pediatr 61:531-545, 1962.

17. Porter IA, Brodie J: Boric acid preservation of urine samples. BMJ 2:353-355, 1969.

18. Fayinka OA: Boric acid: a useful preservative for urine samples. Afr J Med Sci 2:377-385, 1971.

19. Shubair M, Larsen B: Growth inhibition of Candida albicans and other medically important yeasts by vaginal contraceptive products. Gynecol Obstet Invest 29:67$70,1990$.

20. Sobel JD: Vaginitis. N Engl J Med 337:1896-1903, 1997.

21. Nyirjesy P, Seeney SM, Grody MT, et al.: Chronic fungal vaginitis: the value of cultures. Am J Obstet Gynecol 173:820-823, 1995.

22. Spinillo A, Capuzzo E, Gulminetti R, et al.: Prevalence and risk factors for fungal vaginitis caused by nonalbicans species. Am J Obstet Gynecol 176:138-141, 1997.

23. Redondo-Lopez V, Lynch M, Schmitt C, et al.: Torulopsis glabrata vaginitis: clinical aspects and susceptibility to antifungal agents. Obstet Gynecol 76:651-655, 1990.

24. Sobel JD, Vazquez J, Lynch M, et al.: Vaginitis due to Saccharomyces cerevisiae: epidemiology, clinical aspects, and therapy. Clin Infect Dis 16:93-99, 1993.

25. Sobel JD, Vazquez J: Symptomatic vulvovaginitis due to fluconazole-resistant Candida albicans in a female who was not infected with human immunodeficiency virus. Clin Infect Dis 22:726-727, 1996.

26. Skinner GRB, Hartley CE, Millar D, et al.: Possible treatment for cold sores. BMJ 2:704, 1979.

27. Thai L, Hart LL: Boric acid vaginal suppositories. Ann Pharmacother 27:1355-1356, 1993.

28. Spinillo A, Capuzzo E: Fungal vaginitis caused by nonalbicans species. Am J Obstet Gynecol 177:485,1997.

29. Jovanovic R, Congema, E Nguyen HT: Antifungal agents vs. Boric acid for treating chronic mycotic vulvovaginitis. J Reprod Med 36:593-597, 1991.

30. Drug Topics Red Book, 1998: 197. 


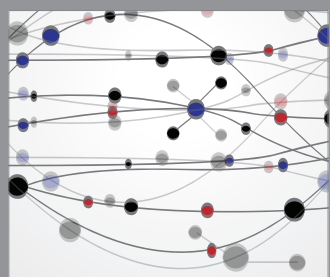

The Scientific World Journal
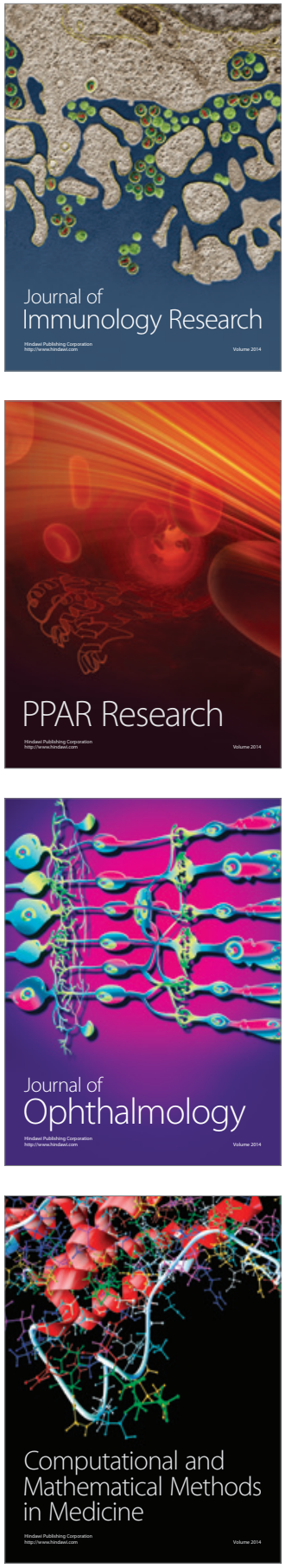

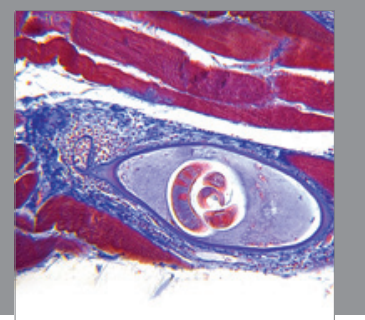

Gastroenterology

Research and Practice
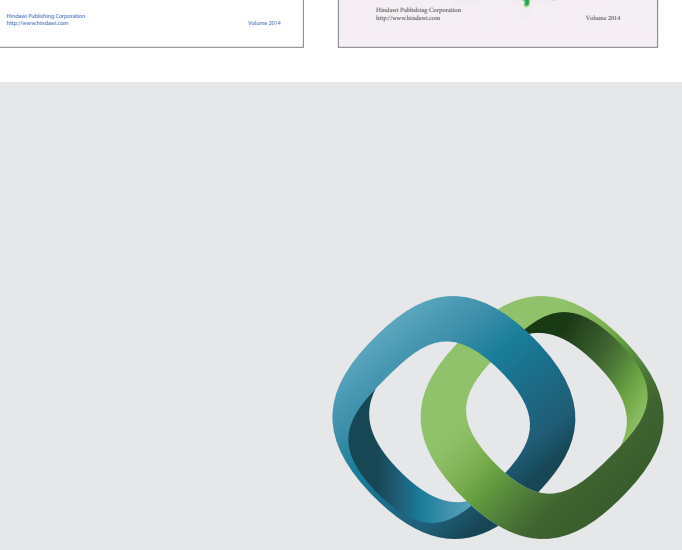

\section{Hindawi}

Submit your manuscripts at

http://www.hindawi.com
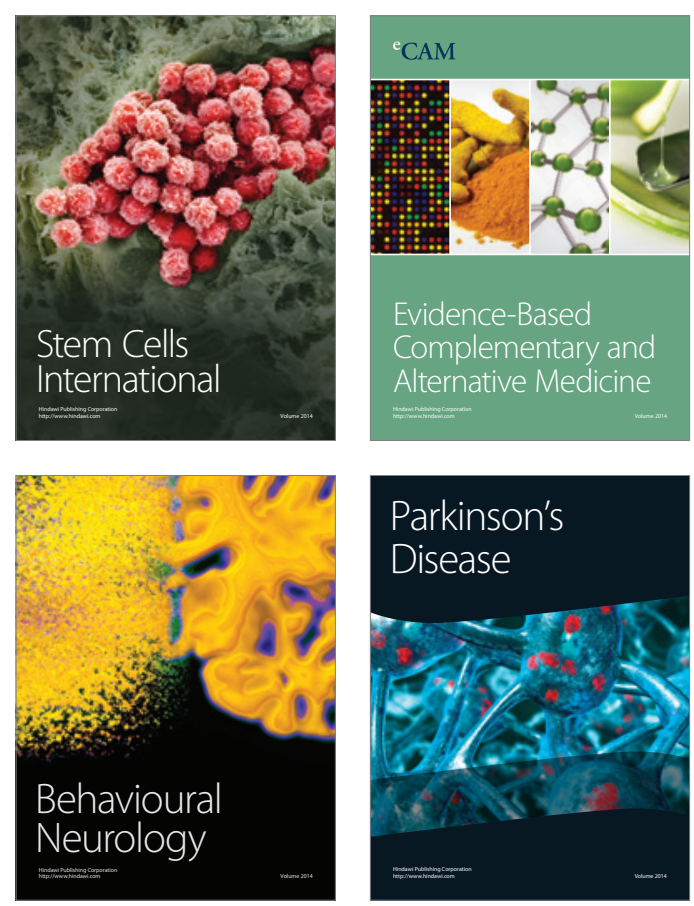

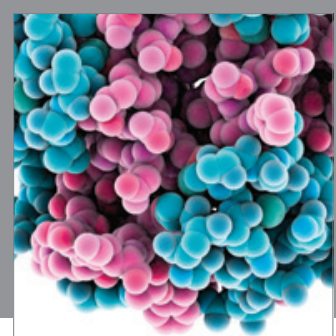

Journal of
Diabetes Research

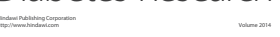

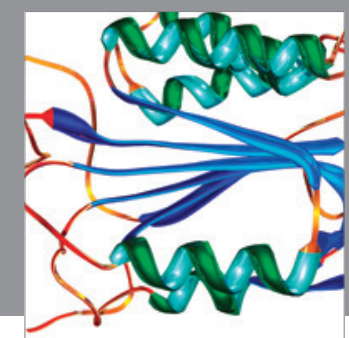

Disease Markers
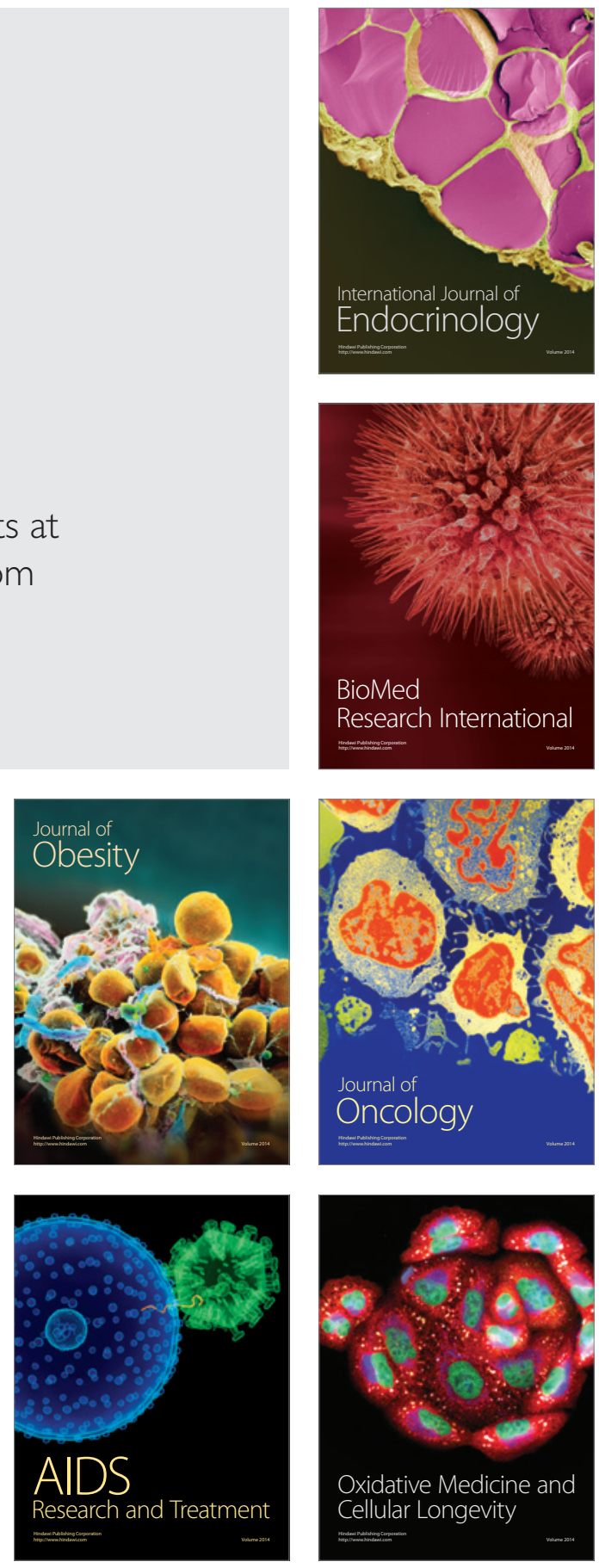